\title{
Concurrent Diagnosis of Acute Myeloid Leukemia and COVID-19: A Management Challenge
}

\author{
Abdul Moiz Khan ${ }^{1}$, Zainub Ajmal ${ }^{1}$, Mihir Raval ${ }^{2}$, Ellis Tobin ${ }^{3}$ \\ 1. Internal Medicine, Albany Medical Center, Albany, USA 2. Hematology and Oncology, Albany Medical Center, \\ Albany, USA 3. Infectious Disease, Upstate Infectious Diseases Associates, Albany, USA
}

Corresponding author: Abdul Moiz Khan, khana13@amc.edu

\begin{abstract}
The emergence of coronavirus disease 2019 (COVID-19) has created new challenges in the management of serious diseases. We describe a 41 -year-old male who presented with fever, watery diarrhea, and epistaxis. Initial workup revealed pancytopenia with $>50 \%$ blasts on the peripheral smear raising suspicion of acute myeloid leukemia (AML) (later confirmed by bone marrow biopsy as AML with myelodysplasia-related changes) and a positive polymerase chain reaction (PCR) for severe acute respiratory syndrome coronavirus 2 (SARS-CoV-2). Given the extraordinary risk, he was treated with remdesivir and convalescent plasma for COVID-19. On admission day 8, repeat PCR for SARS-CoV-2 returned negative and the patient was deemed stable for chemotherapy. Therefore, induction was done with liposomal daunorubicin and cytarabine. However, he did not respond to the therapy and was started on re-induction therapy with decitabine and venetoclax. In our discussion, we review the current principles of treatment of patients with concurrent COVID-19 and AML.
\end{abstract}

Categories: Infectious Disease, Epidemiology/Public Health, Hematology

Keywords: covid 19, novel corona virus, corona virus disease 2019, acute myeloblastic leukemia, acute myeloid leukemia (aml), remdesivir, convalescent plasma therapy

\section{Introduction}

The emergence of coronavirus disease 2019 (COVID-19) caused by severe acute respiratory syndrome coronavirus 2 (SARS-CoV-2) has brought forth new challenges in the management of serious diseases including hematological malignancies. There is a dearth of data on the prognosis and optimal management of patients with concomitant COVID-19 and hematological malignancies [1,2]. In general, some studies suggest that cancer patients may have a higher rate of COVID-19 infection, severe illness, rapid development of severe symptoms and mortality [1-4]. While it is of utmost importance to minimize the risk of COVID-19 in the immunocompromised population, patients require intensive monitoring and modified treatment strategies once the infection occurs.

Received 07/23/2020 Review began $07 / 23 / 2020$ Review ended 07/25/2020 Published 08/09/2020

\section{(c) Copyright 2020}

Khan et al. This is an open access article distributed under the terms of the Creative Commons Attribution License CC-BY 4.0., which permits unrestricted use, distribution, and reproduction in any medium, provided the original author and source are credited.
We describe a patient with newly diagnosed acute myeloid leukemia (AML) and COVID-19, and discuss the current approach of management of such cases.

\section{Case Presentation}

A 41-year-old male with no significant past medical history presented with fever with rigors and chills, fatigue, myalgias, 4-5 episodes daily of watery diarrhea, diffuse abdominal pain and nausea for four days. He also reported one episode of coffee-ground emesis and multiple episodes of epistaxis within the last month. He had some exertional dyspnea but denied cough, chest pain, sore throat, rhinorrhea, anosmia or ageusia. He also denied any travel outside of New York state. On presentation, he had a temperature of a $101 \mathrm{~F}$, tachycardia up to 125 beats/minute, blood pressure at $142 / 65 \mathrm{mmHg}$ and oxygen saturation was $97 \%$ on room air. On physical examination, prominent conjunctival pallor and mild generalized abdominal tenderness were noted. Lungs were clear to auscultation.

Initial workup revealed severe pancytopenia with $>50 \%$ blasts on the peripheral smear raising strong suspicion of acute leukemia, and positive polymerase chain reaction (PCR) for SARS-CoV-2 on nasopharyngeal swab. The patient emergently received intravenous (IV) cefepime and vancomycin for empirical antimicrobial coverage for neutropenic sepsis as well as packed red blood cells and platelet transfusions.

\section{Investigations}

The results of the initial investigations are provided in Table 1. 


\section{Cureus}

\begin{tabular}{|c|c|c|}
\hline Laboratory test & Result & Reference range \\
\hline White blood cells & $2.8 \times 10^{3} \mathrm{cells} / \mathrm{HL}$ & $3.4 \times 10^{3}-10.8 \times 10^{3} \mathrm{celll} / \mathrm{LL}$ \\
\hline Absolute neutrophil count & $0.1 \times 10^{3}$ cells/ $/ \mathrm{LL}$ & $1.5 \times 10^{3}-5.5 \times 10^{3} \mathrm{cells} / \mathrm{LL}$ \\
\hline Absolute Iymphocyte count & $0.3 \times 10^{3}$ cells/ $/ \mathrm{LL}$ & $1.1 \times 10^{3}-3.9 \times 10^{3}$ cells $/ \mathrm{LL}$ \\
\hline Hemoglobin & $5.9 \mathrm{~g} / \mathrm{dL}$ & $11.1-15.9 \mathrm{~g} / \mathrm{dL}$ \\
\hline Hematocrit & $16.4 \%$ & $34-46.6 \%$ \\
\hline Platelets & $12 \times 10^{3} \mathrm{cells} / \mu \mathrm{L}$ & $150 \times 10^{3}-450 \times 10^{3} \mathrm{cells} / \mathrm{hL}$ \\
\hline Prothrombin time & 16.3 seconds & 9.8-11.8 seconds \\
\hline International normalized ratio & 1.4 & 0.83-1.1.14 \\
\hline Fibrinogen & $654 \mathrm{mg} / \mathrm{dL}$ & $172-483 \mathrm{mg} / \mathrm{dL}$ \\
\hline D. dimer & $10.56 \mathrm{mg} / \mathrm{L}$ & $<0.5 \mathrm{mg} / \mathrm{L}$ \\
\hline Lactate dehydrogenase & $527 \mathrm{IU} / \mathrm{L}$ & 90-225 IU/L \\
\hline toglobin & $235.3 \mathrm{mg} / \mathrm{dL}$ & $24-234 \mathrm{mg} / \mathrm{dL}$ \\
\hline Erythrocyte sedimentation rate & $41 \mathrm{~mm} / \mathrm{hr}$ & $0-32 \mathrm{~mm} / \mathrm{hr}$ \\
\hline C-reactive protein & $392.4 \mathrm{mg} / \mathrm{L}$ & $<8 \mathrm{mg} / \mathrm{L}$ \\
\hline Procalcitonin & $27.84 \mathrm{ng} / \mathrm{mL}$ & $<0.5 \mathrm{ng} / \mathrm{mL}$ \\
\hline Ferritin & $1591 \mathrm{ng} / \mathrm{mL}$ & $24-336 \mathrm{ng} / \mathrm{mL}$ \\
\hline Troponin & $0.2 \mathrm{ng} / \mathrm{mL}$ & $0-0.04 \mathrm{ng} / \mathrm{mL}$ \\
\hline Bicarbonate & $19 \mathrm{mmol/L}$ & $21-30 \mathrm{mmol} / \mathrm{L}$ \\
\hline Blood urea nitrogen & $29.3 \mathrm{mg} / \mathrm{dL}$ & $7-22 \mathrm{mg} / \mathrm{dL}$ \\
\hline Creatinine & $1.8 \mathrm{mg} / \mathrm{dL}$ & 0.8-1.4 mg/d/L \\
\hline imated glomerular filtration rate & 49.38 & $>60$ \\
\hline Uric acid & $6.1 \mathrm{mg} / \mathrm{dL}$ & $3.6-8 \mathrm{mg} /$ \\
\hline
\end{tabular}

TABLE 1: Initial investigations

X-ray and computed tomography (CT) scan of the chest were negative for interstitial or lobar pneumonia (Figure 1). CT scan of the abdomen and pelvis showed only mild thickening of small bowel loops.

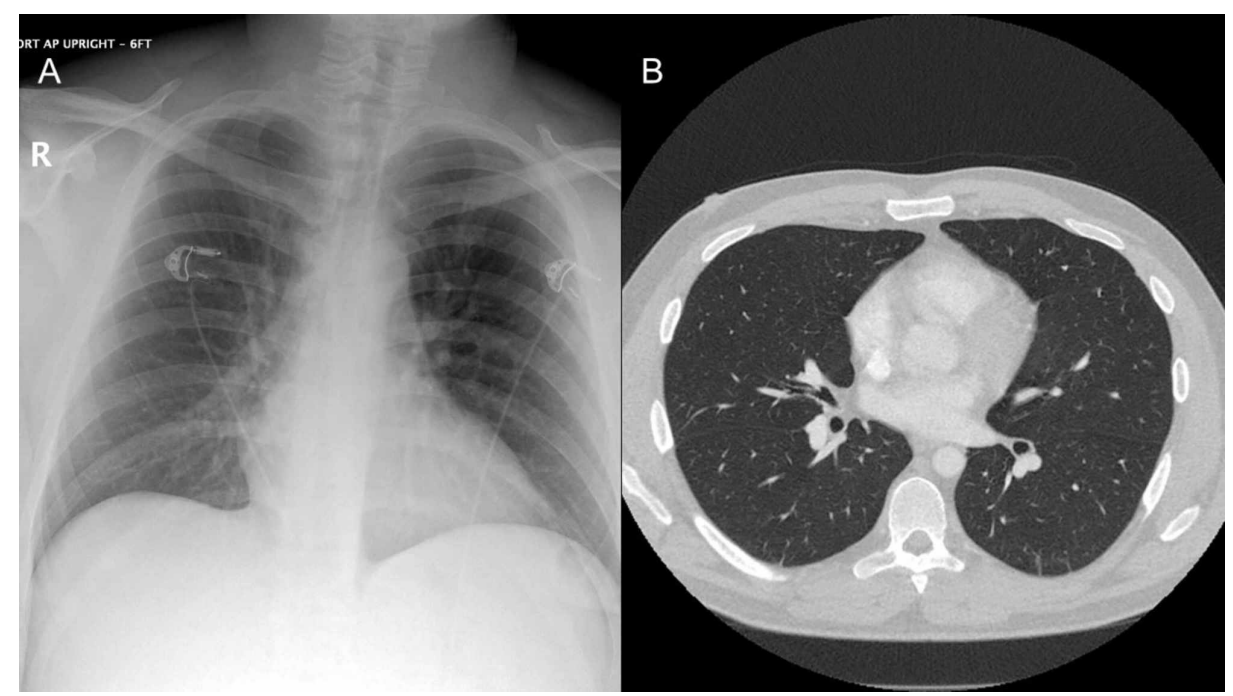


Clostridium difficile toxin gene was detected by PCR in the stool sample. However, enzyme immunoassay (EIA) was negative for the toxin. Urine culture, blood cultures and stool culture were negative.

Peripheral blood flow cytometry revealed $58 \%$ large blasts, positive for cluster of differentiation (CD)117, human leukocyte antigen-DR (HLA-DR), CD34, CD13, CD22 (dim), CD33 (dim, partial), CD56, and CD45 (moderate), and negative for CD11b, CD14, myeloperoxidase (MPO), CD3, CD4, CD5, CD7, CD10, CD19 and CD20.

Bone marrow aspirate was markedly hypercellular with blast population constituting $69 \%$ of marrow cellularity on 500-cell-count differential. Multiparametric flow cytometry analysis of bone marrow aspirate detected 68\% large blasts, positive for CD117, HLA-DR, CD34, CD13, CD22 (dim), CD56, and CD45 (moderate), had a partial dim expression of $\mathrm{CD} 7, \mathrm{CD} 11 \mathrm{~b}$, and $\mathrm{CD} 33$, and were negative for $\mathrm{CD} 14, \mathrm{MPO}, \mathrm{CD} 3$, CD4, CD5, CD10, CD19, CD20, cyCD3 and surface light chains.

Bone marrow biopsy showed hypercellular marrow consisting of sheets of blasts, representing $70 \%-75 \%$ of the overall marrow cellularity. The blasts were highlighted by CD34, CD117, and MPO, and were negative for E-cadherin. FMS-like tyrosine kinase 3 (FLT3) internal tandem duplication (ITD) and FLT3 tyrosine kinase domain mutations were not detected. AML fluorescence in-situ hybridization (FISH) showed $11 \mathrm{q}^{+}, 8 \mathrm{q}^{+}$, and 6p-, myelodysplastic syndrome (MDS) FISH showed 5q- and 8+. Chromosome analysis revealed an abnormal male karyotype with all cells (20/20 cells) exhibiting a complex composite hyperdiploidy karyotype with multiple structural and numerical abnormalities including trisomy 1 , structural rearrangement of $5 q$ that may lead to loss of 5q, trisomy 8, and trisomy 21 . Next-generation sequencing detected a pathogenic frameshift genomic alteration in the TP53 gene (c.636delT; p.R213Dfs*34).

Overall, these findings were consistent with AML with myelodysplasia-related changes (AML-MRC), stratified in the poor/adverse risk category given the 5q deletion, complex karyotype, and TP53 mutation.

\section{Further treatment and outcome}

The patient was managed under the close supervision of hematology and infectious disease specialists. He was treated with remdesivir (first dose $200 \mathrm{mg}$ IV, followed by $100 \mathrm{mg}$ IV daily for four more days) and convalescent plasma for COVID-19. Chemotherapy for AML was delayed to manage COVID-19 appropriately and ensure clinical stability first.

Our patient also tested positive for Clostridium difficile gene but not for toxin. However, given the presence of watery diarrhea, possibility of a false negative result and the overall complexity of the case, oral vancomycin, and IV metronidazole were started. He was also given one dose of IV bezlotoxumab (1000 mg) to reduce the risk of recurrence of Clostridium difficile infection (CDI).

Fortunately, the patient remained stable from the perspective of respiratory system. He also had an appreciable improvement in diarrhea. Packed red blood cell and platelet transfusions were administered as indicated for the anemia and thrombocytopenia. Antimicrobial therapy with cefepime for neutropenic fever, acyclovir for antiviral, and voriconazole for antifungal prophylaxis was continued throughout. Symptomatic care with antipyretics and antiemetics was provided and the patient was closely monitored for DIC and tumor lysis syndrome.

On admission day 8, SARS-CoV-2 real-time PCR was repeated which returned negative. Considering the relative stability and the reassuring negative COVID-19 test, induction therapy for AML was initiated with liposomal daunorubicin $44 \mathrm{mg} / \mathrm{m} 2$ and cytarabine $100 \mathrm{mg} / \mathrm{m} 2$ on days 1,3 , and 5 .

Hospital course after the induction chemotherapy was complicated by recurrent fever and prominent mucositis. Antibiotics were escalated empirically to meropenem and briefly with the addition of IV vancomycin. Patient continued to have profound pancytopenia and $>50 \%$ blasts on the blood smear. Peripheral blood flow cytometry done 14 days after the induction revealed 55\% myeloid blasts suggesting a lack of response to therapy and obviating the need of repeat bone marrow biopsy. Therefore, re-induction therapy with decitabine and venetoclax was started. Prognosis remains poor overall given the adverse risk AML, unresponsiveness to initial chemotherapy and complications of neutropenia.

\section{Discussion}

The concurrence of AML and COVID-19 presents an extraordinary challenge of treating two potentially lifethreatening diseases at the same time. The National Cancer Research Institute (NCRI) AML working group and other expert reviews recommend screening all the patients with AML for SARS-CoV-2 prior to initiation 
of therapy regardless of symptoms [3,5]. Although the induction for newly diagnosed AML is often done on an emergent basis, a reasonable delay in order to test a symptomatic patient for COVID-19, or to provide appropriate management for the COVID-19 infection may be a prudent approach [6]. Therefore, the NCRI AML working group recommends delaying the therapy for AML, if possible, until symptoms resolve and PCR becomes negative [5]. In our patient, we followed this approach of instituting therapy for COVID-19 and obtaining a negative PCR for SARS-CoV-2 prior to induction for the AML. However, a careful determination should be made on a case-by-case basis under expert guidance.

Accurate prognostication of AML with COVID-19 is difficult given the paucity of studies, small sample sizes, and multiple potential confounders. Ferrara et al. have described ten COVID-19 patients with AML, seven of which developed rapid worsening of respiratory function, seven required modifications in the hematological treatment, five died after a median of eight days and death was COVID-19 related in all cases [7]. In addition, Núñez-Torrón et al. have described four patients with AML and COVID-19, three of which developed refractory acute respiratory distress syndrome (ARDS) and eventually died [8]. Of note, none of the patients in these two studies received remdesivir or COVID-19 convalescent plasma. Our patient did not develop a severe respiratory illness from COVID-19 as evident clinically and further substantiated by the imaging. Although it would be hard to assert with certainty, the prompt use of remdesivir and convalescent plasma may have benefitted our patient in that regard.

The choice of treatment regimen for AML depends on the cytogenetic profile and other patient factors. However, general considerations to mitigate the risk of adverse outcomes in AML patients with COVID-19 include reducing the dose of myelosuppressive agents like cytarabine, incorporating less immunosuppressive drugs like venetoclax and azacitidine, decreasing the number of cycles of chemotherapy in select cases, using granulocyte colony stimulating factor (G-CSF) for neutropenia, adding prophylactic antimicrobial agents, and monitoring for drug interactions that may result in adverse effects like QTc prolongation $[5,6,9]$. Allogeneic stem cell transplant should still be pursued if clinically indicated and safe, as for other patients $[5,6]$.

Principles of COVID-19 treatment have been evolving constantly. As of June 16, 2020, there were no Food and Drug Administration (FDA) USA approved drugs for COVID-19 treatment, though various interventions are available through FDA emergency use authorization. Remdesivir has shown benefit in shortening the time to recovery in adults hospitalized with COVID-19 and evidence of lower respiratory tract infection [10]. The National Institute of Health (NIH) USA guidelines recommend remdesivir for the treatment of COVID19 in hospitalized patients with oxygen saturation $\leqslant 94 \%$ on ambient air, and those who require supplemental oxygen, mechanical ventilation or extracorporeal membrane oxygenation (ECMO). Recommended treatment duration is five days in general, and 10 days for patients on mechanical ventilation or ECMO, or who do not have clinical improvement in five days [11]. Furthermore, some studies have reviewed COVID-19 convalescent plasma as an effective therapeutic option [12]. However, the NIH guidelines comment that there is insufficient data to recommend either for or against its use for the treatment of COVID-19 [13]. Although our patient did not have a prominent respiratory illness from COVID19 , the immunosuppression from AML itself and the anticipated chemotherapy posed an exceptional risk of deterioration. Therefore, we pursued with treating our patient with both remdesivir and convalescent plasma.

Another interesting point in our case is the utilization of bezlotoxumab, a monoclonal antibody directed against $\mathrm{C}$. difficile toxin $\mathrm{B}$, indicated as an adjunct treatment for $\mathrm{CDI}$ and associated with a significant reduction in the risk of recurrent CDI over 12 weeks in patients with risk factors for recurrence $[14,15]$. Significant benefits have been demonstrated in patients with one or more of the risk factors that include age $>65$, history of CDI, immunocompromise, or severe CDI [14]. The presence of severe immunocompromise made a strong case for using bezlotoxumab in our patient.

\section{Conclusions}

In the current circumstances, all the patients with AML should be screened for COVID-19 prior to initiation of therapy regardless of symptoms. Chemotherapy for AML may be reasonably delayed in order to provide appropriate management for the COVID-19 infection. Remdesivir and convalescent plasma may be beneficial in some patients although there are no FDA USA approved drugs for COVID-19 to date.

\section{Additional Information}

\section{Disclosures}

Human subjects: Consent was obtained by all participants in this study. Conflicts of interest: In compliance with the ICMJE uniform disclosure form, all authors declare the following: Payment/services info: All authors have declared that no financial support was received from any organization for the submitted work. Financial relationships: All authors have declared that they have no financial relationships at present or within the previous three years with any organizations that might have an interest in the submitted work. Other relationships: All authors have declared that there are no other relationships or activities that could appear to have influenced the submitted work. 


\section{References}

1. Malard F, Genthon A, Brissot E, et al.: COVID-19 outcomes in patients with hematologic disease . Bone Marrow Transplant. 2020, 10.1038/s41409-020-0931-4

2. He W, Chen L, Chen L, et al.: COVID-19 in persons with haematological cancers . Leukemia. 2020, 34:163745. 10.1038/s41375-020-0836-7

3. Paul S, Rausch CR, Jain N, et al.: Treating leukemia in the time of COVID-19. Acta Haematol. 2020, 10.1159/000508199

4. Liang W, Guan W, Chen R, et al.: Cancer patients in SARS-CoV-2 infection: a nationwide analysis in China . Lancet Oncol. 2020, 21:335-7. 10.1016/S1470-2045(20)30096-6

5. AML working party COVID-19 recommendations . (2020). Accessed: June 16, 2020: http://www.cureleukaemia.co.uk/page/news/523/aml-working-party-covid-19-recommendations.

6. Percival MEM, Lynch RC, Halpern AB, et al.: Considerations for managing patients with hematologic malignancy during the COVID-19 pandemic: the Seattle strategy. J Oncol Pract. 2020, 10.1200/OP.20.00241

7. Ferrara F, Zappasodi P, Roncoroni E, Borlenghi E, Rossi G: Impact of Covid-19 on the treatment of acute myeloid leukemia. Leukemia. 2020, 34:2254-2256. 10.1038/s41375-020-0925-7

8. Núñez-Torrón C, García-Gutiérrez V, Tenorio-Núñez MC, Moreno-Jiménez G, López-Jiménez FJ, HerreraPuente P: Poor outcome in patients with acute leukemia on intensive chemotherapy and COVID-19 . Bone Marrow Transplant. 2020, 10.1038/s41409-020-0961-y

9. Gavillet M, Rufer N, Grandoni F, et al.: Hematology in the time of COVID-19 [Article in French]. Rev Med Suisse. 2020, 16:823-6.

10. Beigel JH, Tomashek KM, Dodd LE, et al.: Remdesivir for the treatment of Covid-19 - preliminary report . N Engl J Med. 2020, 10.1056/NEJMoa2007764

11. COVID-19 Treatment Guidelines: potential antiviral drugs under evaluation for the treatment of COVID-19 . (2020). Accessed: June 16, 2020: https://www.covid19treatmentguidelines.nih.gov/antiviral-therapy/..

12. Rajendran K, Krishnasamy N, Rangarajan J, Rathinam J, Natarajan M, Ramachandran A: Convalescent plasma transfusion for the treatment of COVID- 19: systematic review [Epub ahead of print]. J Med Virol. 2020, 10.1002/jmv.25961

13. COVID-19 Treatment Guidelines: immune-based therapy under evaluation for treatment of COVID-19 . (2020). Accessed: May 12, 2020: https://www.covid19treatmentguidelines.nih.gov/immune-based-therapy/..

14. Johnson S, Gerding DN: Bezlotoxumab. Clin Infect Dis. 2019, 68:699-704. 10.1093/cid/ciy577

15. Wilcox MH, Gerding DN, Poxton IR, et al.: Bezlotoxumab for prevention of recurrent Clostridium difficile infection. N Engl J Med. 2017, 376:305-17. 10.1056/NEJMoa1602615 